\title{
Stress oscillations and surface alloy formation during the growth of $\mathrm{FeMn}$ on $\mathrm{Cu}(001)$
}

\author{
Wei Pan, ${ }^{1,2}$ Dirk Sander, ${ }^{2}$ Minn-Tsong Lin, ${ }^{1, *}$ and Jürgen Kirschner ${ }^{2}$ \\ ${ }^{1}$ Department of Physics and Center for Nanostorage Research, National Taiwan University, Taipei 106, Taiwan \\ ${ }^{2}$ Max-Planck-Institut für Mikrostrukturphysik, Weinberg 2, D-06120 Halle, Germany
}

(Received 16 May 2003; published 19 December 2003)

\begin{abstract}
In situ stress and medium-energy electron-diffraction (MEED) measurements have been performed simultaneously during the deposition of FeMn on $\mathrm{Cu}(001)$. For a thickness above 5 layers, stress and MEED exhibit coherent layer-by-layer oscillations with a period of one atomic layer, where the largest compressive stress corresponds to the filled layer. In this thickness regime, the average stress is $-0.59 \pm 0.02 \mathrm{GPa}$. From this, we deduce the biaxial modulus of FeMn layers as $148( \pm 5) \mathrm{GPa}$, which agrees well with the respective bulk value. For a FeMn thickness below 1.5 layers, the resulting stress is qualitatively ascribed to the sum of the individual stress contributions from $\mathrm{Fe}$ on $\mathrm{Cu}(001)$ and $\mathrm{Mn}$ on $\mathrm{Cu}(001)$. $\mathrm{A} c(2 \times 2)$ low-energy electron diffraction pattern in this thickness regime indicates the formation of a $c(2 \times 2) \mathrm{MnCu}$ surface alloy in the initial growth of $\mathrm{FeMn}$ on $\mathrm{Cu}(001)$, which induces a compressive surface stress of $-0.7 \mathrm{~N} / \mathrm{m}$ for the initial deposition of the FeMn alloy. This surface alloy formation leads to a Fe-rich FeMn alloy near the $\mathrm{Cu}$ interface. This compositional change might modify the antiferromagnetic coupling of the 1:1 FeMn alloy.
\end{abstract}

DOI: 10.1103/PhysRevB.68.224419

PACS number(s): 75.70.-i, 68.35.Gy, 68.35.Ct, 68.55.-a

\section{INTRODUCTION}

$\mathrm{Fe}_{x} \mathrm{Mn}_{1-x}$ alloys are prototypes for antiferromagnetic materials with a high Néel temperature $T_{N} \cdot{ }^{1}$ FeMn thin films are widely used in a spin-valve structure in combination with magnetoresistive materials for application in magnetic sensors and magnetic data storage. ${ }^{2}$ The lattice constant of the $\mathrm{Fe}_{x} \mathrm{Mn}_{1-x}$ alloys us depends on the alloy composition $x .{ }^{1}$ This allows to choose a composition $x$, which leads to a moderate misfit between alloy film and substrate. For the 1:1 composition, i.e., $x=0.5$, the lattice constant of bulk FeMn is $3.629 \AA^{1}{ }^{1}$ This alloy has a face-centered cubic structure and its deposition on $\mathrm{Cu}(001)$ could lead to epitaxially ordered films, as the misfit between $\mathrm{FeMn}$ and $\mathrm{Cu}$ is small and it amounts to $\eta=\left(a_{\mathrm{Cu}}-a_{\mathrm{FeMn}}\right) /\left(a_{\mathrm{FeMn}}\right)=-0.4 \%$.

This paper focuses on combined stress measurements and diffraction experiments to correlate atomic structure and resulting stress in the thickness range from 0 to 18 layers of FeMn on $\mathrm{Cu}(001)$. We find that in contrast to a simplistic growth model where FeMn alloy layers grow pseudomorphically strained on $\mathrm{Cu}(001)$, the formation of a surface alloy between $\mathrm{Mn}$ and $\mathrm{Cu}$ is clearly observed by the stress and diffraction experiments. Only for an alloy thickness above 5 layers, we find experimental evidence from stress oscillations that the alloy grows in a layer-by-layer fashion. The formation of the $\mathrm{MnCu}$ surface alloy has important consequences for the magnetic properties of ultrathin FeMn films, as due to the surface alloy formation, $\mathrm{Mn}$ atoms are incorporated into the $\mathrm{Cu}$ surface. This intermixing might affect the antiferromagnetic coupling near the FeMn-Cu interface.

\section{EXPERIMENT}

The experiment was performed in an ultrahigh vacuum chamber with a base pressure of $1 \times 10^{-10}$ mbar. A $124 \mu \mathrm{m}$ thin $\mathrm{Cu}(001)$ crystal (width: $3 \mathrm{~mm}$, length: $12 \mathrm{~mm}$ ) was used as a substrate. The long edge of the crystal was oriented along the [110] direction. It was clamped at one end along its width, and the other end was free. This mounting allows us to measure the stress during FeMn deposition by measuring the change of curvature of the crystal during film growth. ${ }^{3}$ The surface of the $\mathrm{Cu}(001)$ was cleaned by $\mathrm{Ar}^{+}$sputtering and followed by short annealing to $720 \mathrm{~K}(30 \mathrm{~s})$ by radiation from a $\mathrm{W}$ shield, which was heated by a $W$ filament. The surface cleanliness was checked by Auger electron spectroscopy (AES) and sharp diffraction spots of low-energy electron-diffraction (LEED) indicated good crystalline ordering. The FeMn alloy was grown by codeposition from individual $\mathrm{Fe}$ and $\mathrm{Mn}$ evaporators. ${ }^{4,5}$ The purity of $\mathrm{Mn}$ and $\mathrm{Fe}$ is 99.5\% and $99.99 \%$ (at.\%), respectively. The deposition rate of the alloy was set to a 1:1 atomic ratio by adjusting the evaporation rate of each evaporator, as confirmed by individual MEED measurements and by AES. ${ }^{6-9}$ The error for the alloy composition is estimated to be $\pm 5 \%$. Both evaporators were adjusted to give a deposition rate close to 0.25 $\mathrm{ML} / \mathrm{min}$, which results in a growth rate of the FeMn alloy of $0.5 \mathrm{ML} / \mathrm{min}$. Here ML stands for monolayer.

The stress measurement was carried out during film growth at room temperature. The mechanical stress in the alloy film $\tau_{f}$ induces a change of curvature of the crystal $\Delta(1 / R), \Delta\left(\tau_{f} t_{f}\right)=\left[Y t_{s}^{2} / 6(1-\nu)\right] \Delta(1 / R)$, where $t_{f}$ and $t_{s}$ are the thicknesses of the alloy film (FeMn) and the substrate $(\mathrm{Cu})$, respectively, $Y$ and $\nu$ are the Young's modulus and Poisson ratio of $\mathrm{Cu}(001)$, and $\mathrm{R}$ is the radius of the curvature. $^{10,11}$ The slope of the curvature signal as a function of film thickness gives the film stress $\tau_{f}$ in gigapascal. The curvature was obtained by a highly sensitive optical beam deflection technique, which is schematically shown in Fig. 1. Two laser beams are reflected from two points of the surface, which are separated by a distance $d$, onto two positionsensitive split-photodiode detectors. The detectors are mounted at a distance $L$ away from the substrate. Thus the curvature $\Delta(1 / R)$ is derived from the difference of the position signal as $\Delta(1 / R)=\left(\Delta P_{1}-\Delta P_{2}\right) / 2 d L$, where $\Delta P_{1}$ and $\Delta P_{2}$ are the changes of the position signals for the bottom 


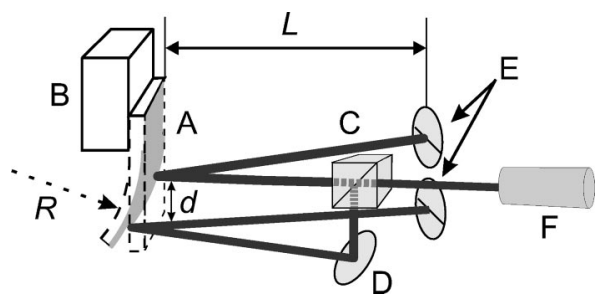

FIG. 1. Schematic representation of the two-beam optical deflection setup to measure the stress-induced $\mathrm{Cu}(001)$ curvature. $A$ : $\mathrm{Cu}(001)$ substrate, $B$ : sample holder, $C$ : beam splitter, $D$ : mirror, $E$ : position sensitive detectors, $F$ : laser, $L$ : distance from substrate to detectors $(\approx 200 \mathrm{~mm}), R$ : radius of $\mathrm{Cu}(001)$ curvatu, and $d$ : distance between two spots $(\approx 4 \mathrm{~mm})$.

and the top signal. For further details of the curvature technique see Ref. 10.

In addition to the stress measurements, film growth was also characterized by monitoring the specular $(0,0)$ intensity of medium-energy electron diffraction (MEED) during deposition ( $3 \mathrm{keV}$ electron beam energy, angle of incidence $3^{\circ}$ in the [110] in-plane azimuth). Oscillations of the specular MEED intensity as a function of deposition time indicate the filling of individual FeMn layers. ${ }^{5,12}$ The surface structure was characterized by LEED.

\section{RESULTS}

\section{A. Stress and MEED of FeMn on $\mathrm{Cu}(001)$ during deposition}

Both stress and MEED were measured simultaneously during deposition of $\mathrm{FeMn}$ on $\mathrm{Cu}(001)$. The results are plotted as a function of deposition time in Fig. 2. The MEED

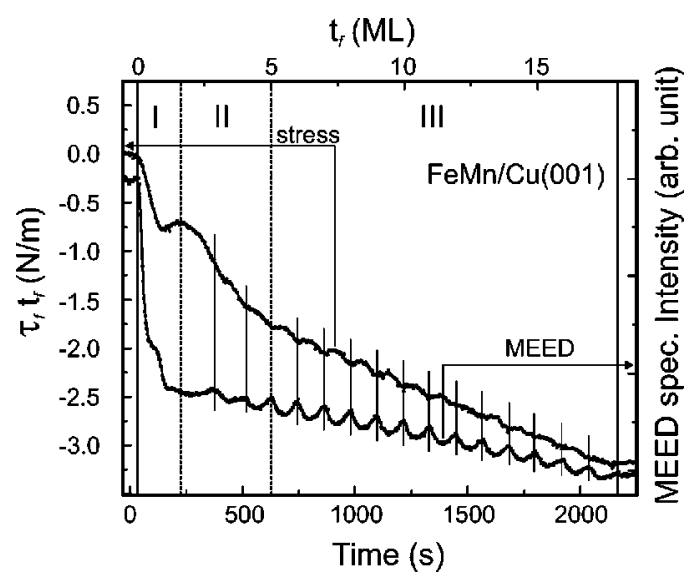

FIG. 2. Stress and MEED as a function of deposition time for the alloy $\mathrm{FeMn}$ on $\mathrm{Cu}(001)$. Three stress regimes are identified: (I) interface formation below 1.5 ML, (II) between 1.5 and $5 \mathrm{ML}$, compressive stress of $1.87 \mathrm{GPa}$ is found but obvious MEED oscillations are absent, and (III) layer-by-layer mode above $5 \mathrm{ML}$, where stress and MEED oscillate with a period of $1 \mathrm{ML}$. The minimum of the compressive stress coincides with the maximum of the MEED intensity, which indicates the largest compressive stress for a filled layer ( 1 layer $=1 \mathrm{ML}: 1.5 \times 10^{-15}$ atoms $/ \mathrm{cm}^{2}$ ). The vertical lines indicate the coherence between stress and MEED oscillations. A negative slope of the stress curve indicates compressive stress and a positive slope indicates tensile stress. data do not show any oscillations for the deposition of the first and the second layer, instead a drop of reflected intensity is observed. The oscillatory behavior of the MEED specular intensity starts at the third layer, and it is well pronounced after the fifth layer $\left(1\right.$ layer $=1 \mathrm{ML} \simeq 1.82 \AA \mathrm{A}: 1.5 \times 10^{15}$ atoms $/ \mathrm{cm}^{2}$ ).

The stress behavior of $\mathrm{FeMn}$ on $\mathrm{Cu}(001)$ is characterized by three regimes. In regime I (thickness below 1.5 ML) the negative slope of the stress curve up to $1 \mathrm{ML}$ indicates initially compressive stress, which is followed from 1.0 ML to 1.5 ML by tensile stress. In this regime, the stress is similar (but not identical) to the calculated sum of the stresses as derived from individual depositions of $\mathrm{Fe}$ on $\mathrm{Cu}(001)$ and $\mathrm{Mn}$ on $\mathrm{Cu}(001)$, as will be discussed in detail in the following section.

In regime II (1.5 ML-5 ML), the thickness integrated stress $\tau_{f} t_{f}$ remains compressive, and it increases in magnitude from -0.7 to $-1.8 \mathrm{~N} / \mathrm{m}$. The film stress $\tau_{f}$ as given by the slope of the curve is $-1.9( \pm 0.2)$ GPa. Stress oscillations are observed for $t_{f}>3 \mathrm{ML}$, and they are coherent with the MEED intensity oscillations as indicated by the vertical lines. In regime III, (thickness above $5 \mathrm{ML}$ ), the stress performs pronounced layer-by-layer oscillations, as does the MEED specular intensity. The sequence of local minima of the stress curve coincides with the maxima of the MEED specular intensity, i.e., the stress oscillates with a period of 1 ML. The largest compressive stress, i.e., the minimum of the stress curve, corresponds to the filled layer (MEED maximum). The average slope of the stress curve indicates a film stress of $\tau_{f}=-0.59( \pm 0.02) \mathrm{GPa}$ in this regime, which is ascribed to the epitaxial misfit between $\mathrm{FeMn}$ and $\mathrm{Cu}$, as discussed below.

\section{B. Stress behavior of $\mathrm{Fe}$ on $\mathrm{Cu}(001)$, $\mathrm{Mn}$ on $\mathrm{Cu}(001)$, and $\mathrm{FeMn}$ on $\mathrm{Cu}(001)$ at low coverage}

In order to clarify the origin of distinct stress regimes for the FeMn alloy deposition as depicted in Fig. 2, we performed also stress measurements during the deposition of the individual alloy components $\mathrm{Fe}$ and $\mathrm{Mn}$ on $\mathrm{Cu}(001)$, and we present the results in Fig. 3. The top curve of Fig. 3(a) indicates an almost stress free growth of $\mathrm{Fe}$ on $\mathrm{Cu}(001)$ up to 0.5 ML, which is followed by tensile stress $\tau_{f} t_{f}$ of $0.7 \mathrm{~N} / \mathrm{m}$ at 2 ML. The lower curve of Fig. 3(a) indicates the Mn-induced stress on $\mathrm{Cu}(001)$. Mn induces compressive stress which grows in proportion to the deposited amount until at $0.5 \mathrm{ML}$ $\mathrm{Mn}$, a stress $\tau_{f} t_{f}$ of $-1.2 \mathrm{~N} / \mathrm{m}$ is measured. Further deposition leads to slightly enhanced compressive stress which levels off at $\tau_{f} t_{f}=-1.4 \mathrm{~N} / \mathrm{m}$ for $2 \mathrm{ML} \mathrm{Mn}$.

In Fig. 3(b), we compare the measured stress for the FeMn alloy (filled circles) with the calculated sum (solid line) from the individual stress curves for Fe and Mn shown in (a). We rescaled the thickness axis for the calculated stress curve by a factor of 2 , such that $1 \mathrm{ML} F \mathrm{FeMn}$ on $\mathrm{Cu}(001)$ is compared to the deposition of $0.5 \mathrm{MLFe}$ on $\mathrm{Cu}(001)$ and 0.5 ML Mn on $\mathrm{Cu}(001)$.

Below 1.5 ML, the calculated and alloy-induced stress shows a comparable thickness dependence. The calculated sum signal and the alloy-induced stress both lead to a com- 


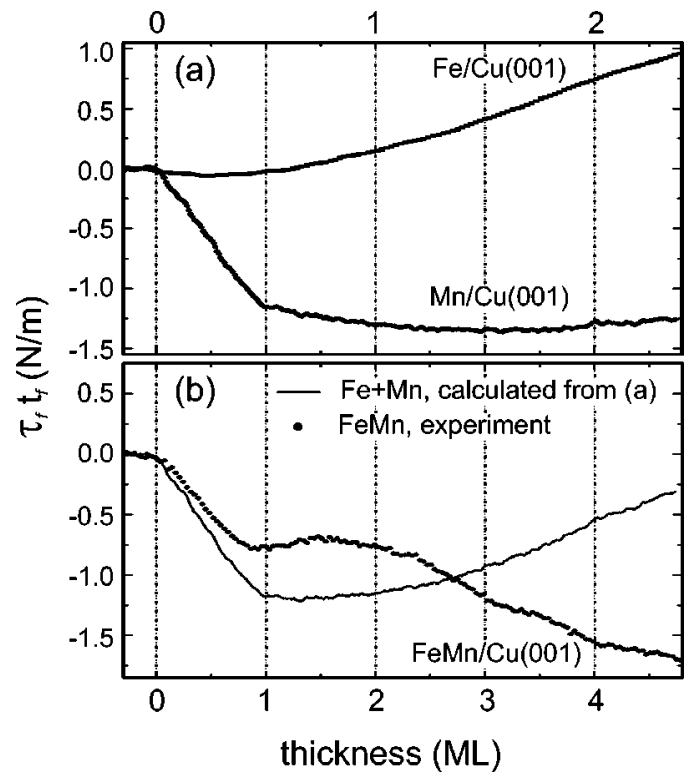

FIG. 3. Stress as a function of thickness for different deposits: (a) individual stress measurement for $\mathrm{Fe}$ on $\mathrm{Cu}(001)$ and $\mathrm{Mn}$ on $\mathrm{Cu}(001)$. (b) Calculated stress as the sum of $\mathrm{Fe}$ on $\mathrm{Cu}(001)$ and $\mathrm{Mn}$ on $\mathrm{Cu}(001)$ from (a), and stress for the deposition of the alloy FeMn on $\mathrm{Cu}(001)$. The stress for the alloy $\mathrm{FeMn}$ on $\mathrm{Cu}(001)$ is not given simply by the sum of the ingredients. The alloy leads to compressive stress for $t_{f}>2 \mathrm{ML}$, whereas the calculated sum of the individual components leads to a tensile stress.

pressive stress which levels off after the deposition of $1 \mathrm{ML}$. The magnitude of the induced compressive stress is however smaller by a factor of 0.63 for the alloy-induced stress as compared to the individual stress contributions. The similar compressive stress behavior in the low coverage regime for $\mathrm{FeMn}$ on $\mathrm{Cu}(001)$ and $\mathrm{Mn}$ on $\mathrm{Cu}(001)$ indicates that a surface alloy might also form in the initial growth of FeMn on $\mathrm{Cu}(001)$.

However, above 1.5 ML, the calculated stress showed an opposite tendency as compared to the measured alloyinduced stress, which was compressive and oscillatory. This implies that the stress behavior is determined by the alloy of the deposits and not by the sum of the individual components for an alloy thickness above 1.5 ML.

\section{LEED investigation of the Fe, Mn, and FeMn deposits on $\mathrm{Cu}(001)$}

To clarify the formation of the surface alloy in the low coverage regime of the deposition of $\mathrm{FeMn}$ on $\mathrm{Cu}(001)$, LEED was used to characterize the $c(2 \times 2)$ pattern, which is due to the surface alloy of $\mathrm{MnCu} .^{13}$ This $c(2 \times 2)$ pattern is ascribed to the ordered buckling of the surface atoms of the alloy, as proposed by many previous experimental (scanning tunnel microscope and LEED) and theoretical studies in Refs. 13-18. The LEED patterns for deposition of 0.5 ML Fe on $\mathrm{Cu}(001), 0.5 \mathrm{ML} \mathrm{Mn}$ on $\mathrm{Cu}(001)$, and $1 \mathrm{ML}$ FeMn on $\mathrm{Cu}(001)$ are shown in Figs. 4(a), 4(b), and 4(c), respectively. The $c(2 \times 2)$ pattern is observed for both depositions, $1 \mathrm{ML}$ FeMn on $\mathrm{Cu}(001)$ [Fig. 4(c)], and 0.5 ML Mn on $\mathrm{Cu}(001)$ [(Fig. 4(b)]. This may indicate the formation of the same

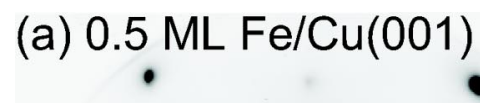

(01)

(10)

\section{(b) $0.5 \mathrm{ML} \mathrm{Mn/Cu(001)}$}

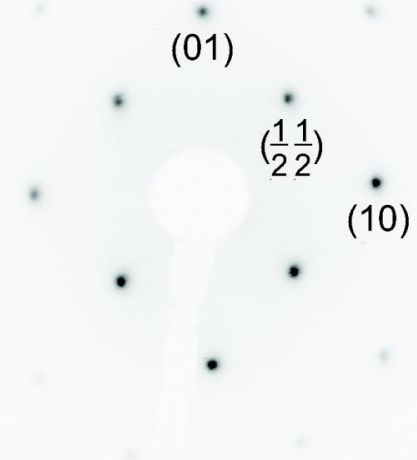

(c) $1 \mathrm{ML} \mathrm{FeMn/Cu(001)}$

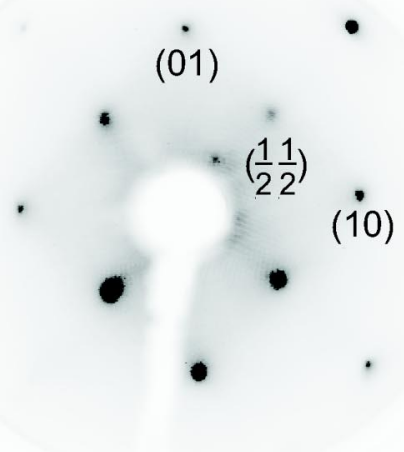

FIG. 4. LEED pattern taken at $112 \mathrm{eV}$ for different deposits: (a) 0.5 ML Fe on $\mathrm{Cu}(001)$, (b) 0.5 ML Mn on $\mathrm{Cu}(001)$, and (c) $1 \mathrm{ML}$ FeMn on $\mathrm{Cu}(001)$. The $c(2 \times 2)\left[\left(\frac{1}{2} \frac{1}{2}\right)\right]$ diffraction pattern was found both in (b) and (c), but not in (a). Mn forms a $c(2 \times 2) \mathrm{MnCu}$ surface alloy in $\mathrm{FeMn}$ on $\mathrm{Cu}(001)$ that obtained for the deposition of $\mathrm{Mn}$ on $\mathrm{Cu}(001)$, which gives rise to the same diffraction images.

$c(2 \times 2) \mathrm{MnCu}$ surface alloy in both cases. No $c(2 \times 2)$ pattern is observed for $0.5 \mathrm{ML} \mathrm{Fe}$ on $\mathrm{Cu}(001)$ [Fig. 4(a)].

The authors in Ref. 18 indicate that the $\mathrm{Mn}-\mathrm{Cu}$ binding energy, which is stronger than Mn-Mn binding energy, leads to $\mathrm{MnCu}$ alloy formation through an incorporation mechanism at step edges. In our case, the FeMn layer was grown in a way of codeposition. Since $\mathrm{Mn}-\mathrm{Cu}$ may have a stronger binding energy than $\mathrm{Fe}-\mathrm{Mn}$ or $\mathrm{Fe}-\mathrm{Cu}$, the $\mathrm{Mn}-\mathrm{Cu}$ alloy formation at very initial growth stage becomes thus mostly fa- 
vored. Further experimental work for a direct evidence of $\mathrm{MnCu}$ alloy formation, such as scanning tunnel microscope observation, is ongoing.

\section{DISCUSSION}

The stress measurements during growth of FeMn on $\mathrm{Cu}(001)$ show a complex dependence on film thickness. Both stress measurements and MEED indicate that a simplistic layer-by-layer growth model is not appropriate for films thinner than $5 \mathrm{ML}$. In thicker films, however, layer-by-layer growth is supported by our measurements, and we start with the discussion of this thickness regime, before we elucidate the decisive role of surface alloy formation between $\mathrm{Mn}$ and $\mathrm{Cu}$ for thinner films.

FeMn films thicker than 5 ML show pronounced stress oscillations and oscillations of the reflected specular MEED intensity with a period of one monolayer, as indicated in regime III of Fig. 2. The coherence between MEED and stress oscillations indicates the strongest compressive stress in the growing film when the layer is filled. In the initial phase of layer filling, the stress does not change significantly, whereas for the completion of the layer filling, the stress change is much more pronounced. This behavior is ascribed to the stress relaxation in FeMn islands, which cover the surface for the initial layer filling. Stress relaxation is most efficient for small islands, whereas it is negligible in the later stage of layer filling, where larger islands are formed and individual islands coalesce. Such oscillatory stress relaxations are a common phenomenon during island growth of epitaxially strained systems, ${ }^{19}$ and here they indicate that the FeMn alloy can be treated as a deposit with a stress behavior which is given by the misfit between FeMn and $\mathrm{Cu}(001)$.

The average stress in the thickness regime III is given by the slope of the stress curve as $\tau_{f}=-0.59 \pm 0.02 \mathrm{GPa}$. The misfit between FeMn and $\mathrm{Cu}$ is $\eta=-0.4 \%$, and we derive a biaxial modulus of the FeMn film as $Y /(1-\nu)=\tau_{f} / \eta$ $=148 \pm 5 \mathrm{GPa}$. This derived biaxial modulus agrees well with the value of $155 \mathrm{GPa}$, as measured by ultrasonic pulseecho-overlap technique for a bulk $\mathrm{Fe}_{0.6} \mathrm{Mn}_{0.4}$ alloy. ${ }^{20}$ We conclude that the stress in regime III can be quantitatively ascribed to the epitaxial misfit between FeMn and $\mathrm{Cu}$. This suggests that FeMn films thicker than 5 ML behave elastically like the respective bulk material.

However, in regime I for a thickness of up to $1.5 \mathrm{ML}$, the stress behavior of the FeMn alloy resembles qualitatively that of the deposit Mn alone. We conclude that the stress in the low coverage regime is mainly due to the surface alloy formation between $\mathrm{Mn}$ and $\mathrm{Cu}$. The observation of a compressive stress due to the surface alloy formation between $\mathrm{Mn}$ and $\mathrm{Cu}$ seems plausible due to the larger atomic size of $\mathrm{Mn}$ as compared to $\mathrm{Cu} .^{21,22}$ The insertion of the larger size of $\mathrm{Mn}$ atoms could induce compressive stress. We cannot exclude that also Fe favors the formation of a surface alloy with $\mathrm{Cu}$, however, in contrast to $\mathrm{Mn}$, no specific alloyinduced super structure is observed by LEED in the submonolayer regime. One might speculate that the almost stress-free growth of $\mathrm{Fe}$ on $\mathrm{Cu}(001)$ up to $0.5 \mathrm{ML} \mathrm{Fe}$ is due to the compensating effect of the tensile strain in epitaxially strained Fe, ${ }^{19}$ which should lead to tensile stress, and a compressive stress due to a possible surface alloy formation of $\mathrm{FeCu}$.

The treatment of coevaporated Fe and $\mathrm{Mn}$ as a FeMn alloy is not appropriate for the initial growth. In this initial thickness regime, the tendency of $\mathrm{Mn}$, and possibly $\mathrm{Fe}$, to form a surface alloy with $\mathrm{Cu}$ is decisive for the elemental composition near the $\mathrm{Cu}$ interface. It should be noted that the stress for codeposition of $\mathrm{Fe}$ and $\mathrm{Mn}$ on $\mathrm{Cu}(001)$ is smaller by a factor of 0.63 than the calculated stress of the individual deposits $\mathrm{Fe}$ on $\mathrm{Cu}(001)$ and $\mathrm{Mn}$ on $\mathrm{Cu}(001)$. This deviation of the stress measurement below $1 \mathrm{ML}$ of $\mathrm{FeMn} / \mathrm{Cu}(100)$ from that of $\mathrm{Mn} / \mathrm{Cu}(100)$ would be simply explained by considering a partial incorporation of $\mathrm{Fe}$ atoms into the $\mathrm{MnCu}$ surface alloy, which is increasing with the deposition time. This leads to a dilution of the Mn solutes by the incorporation of $\mathrm{Fe}$, and in turn to a reduction of the stress of the $\mathrm{FeMn} / \mathrm{Cu}(100)$ as compared to that of $\mathrm{Mn} / \mathrm{Cu}(100)$ or the sum of those of $\mathrm{Mn} / \mathrm{Cu}(100)$ and $\mathrm{Fe} / \mathrm{Cu}(100)$.

The picture for epitaxial growth with codeposition could also be applied in other alloy film systems. Once one of the deposited elements has the tendency to form an alloy with the substrate, the first few layers should not be considered as an alloy with a composition given by the respective deposition rates. Rather, a possible surface alloy formation between deposit and substrate might lead to an alloy composition, which varies with distance from the interface. Near the interface, the deviation from the stoichiometry as intended from the deposition rates will be the largest, and the intended alloy composition will be found only in a distance of a few atomic layers away from the interface. From our stress measurements we deduce a distance of approximately $5 \mathrm{ML}$, which is necessary to achieve the nominal alloy composition.

In this study, the surface alloy formation between Mn and $\mathrm{Cu}$ may have important implications for the magnetic properties near the FeMn-Cu interface. Near the interface, the alloy will be not a 1:1 FeMn layer, as Mn forms readily a $c(2 \times 2) \mathrm{MnCu}$ surface alloy. One might suspect that this compositional change could effect the antiferromagnetic coupling of FeMn near the FeMn-Cu interface.

In addition to intermixing due to the surface alloy formation, the contact with a ferromagnetic layer in an exchangebiased bilayer system can also induce the change of magnetic coupling or a net magnetic moment of the FeMn at a bilayer interface. Both our previous theoretical ${ }^{23}$ and experimental ${ }^{24}$ studies showed that the induced net moment was confined to the interfacial alloy layers and strongly varied with FeMn thickness.

The formation of the $\mathrm{MnCu} c(2 \times 2)$ surface alloy has important consequences for the subsequent growth of FeMn. This growth does not occur on a FeMn layer, but rather on a chemically and presumably also structurally inhomogeneous FeCuMn surface. The $c(2 \times 2)$ structure of the $\mathrm{MnCu}$ alloy suggests a rougher surface with enhanced corrugation as compared to a flat surface, and this will affect the diffusion of the deposited species, and thereby also the resulting film morphology in regime II. This impact of the surface alloy formation on the film morphology is corroborated by the 
absence of MEED oscillations of a thickness up to $2 \mathrm{ML}$, which indicates a rougher surface in this thickness range.

\section{CONCLUSION}

Compressive stress and the $c(2 \times 2)$ LEED pattern indicate a surface alloy formation in the initial growth of FeMn on $\mathrm{Cu}(001)$. It affects the chemical composition of the first three monolayers ML, and might also affect the antiferromagnetic coupling of the FeMn film near the interface with $\mathrm{Cu}$ substrate. The coherent stress and MEED specular intensity oscillations above $5 \mathrm{ML}$ indicate that FeMn on $\mathrm{Cu}(001)$ grows in the layer-by-layer mode in thicker films. This study suggests that a surface alloy formation can be detected by the respective stress signature. The impact of a surface alloy formation on the physical properties, e.g., structure, and magnetic property, is particularly important for the growth of alloys, where those physical properties often depend critically on the alloy composition.

\section{ACKNOWLEDGMENTS}

We thank H. Menge for the skillful preparation of the $\mathrm{Cu}(001)$ crystal. This work was partially supported by NSC, MOE excellent program, and MOEA (92-EC-17-A-08-S10006) of Taiwan. M.-T. Lin and W. Pan acknowledge the financial support from the Max-Planck-Society of Germany.
*Author to whom correspondence should be addressed. Email address: mtlin@phys.ntu.edu.tw

${ }^{1}$ Y. Endoh and Y. Ishikawa, J. Phys. Soc. Jpn. 30, 1614 (1971).

${ }^{2}$ J. Nogués and I.K. Schuller, J. Magn. Magn. Mater. 192, 203 (1999).

${ }^{3}$ D. Sander, Rep. Prog. Phys. 62, 809 (1999).

${ }^{4}$ C.C. Kuo, W. Pan, Y.C. Chen, and M.-T. Lin, J. Appl. Phys. 93, 8743 (2003).

${ }^{5}$ F. Offi, W. Kuch, and J. Kirschner, Phys. Rev. B 66, 064419 (2002).

${ }^{6}$ M.-T. Lin, W.C. Lin, C.C. Kuo, and C.L. Chiu, Phys. Rev. B 62, 14268 (2000)

${ }^{7}$ W.C. Lin, C.C. Kuo, C.L. Chiu, and M.-T. Lin, J. Appl. Phys. 89, 7139 (2001).

${ }^{8}$ W.C. Lin, C.C. Kuo, C.L. Chiu, and M.-T. Lin, Surf. Sci. 478, 9 (2001).

${ }^{9}$ C.C. Kuo, S.F. Chuang, W. Pan, W.C. Lin, and M.-T. Lin, J. Appl. Phys. 91, 7185 (2002).

${ }^{10}$ H. Ibach, Surf. Sci. Rep. 29, 193 (1997).

${ }^{11}$ R.F.S. Hearmon, in The Elastic Constants of Crystals and Other Anisotropic Materials, edited by K.-H. Hellwege and A.M. Hellwege (Landolt-Börnstein New-Series, Group III), Vol. 18, supplement to III/11 (Springer, Berlin, 1984) $[Y /(1-\nu)]_{\mathrm{Cu}}$ $=114 \mathrm{GPa}$.

${ }^{12}$ W. Pan, N.Y. Jih, Y.C. Chen, C.H. Chuang, C.C. Kuo, and M.-T.
Lin (unpublished).

${ }^{13}$ T. Flores, M. Hansen, and M. Wuttig, Surf. Sci. 279, 251 (1992).

${ }^{14}$ M. Wuttig, Y. Gauthier, and S. Blügel, Phys. Rev. Lett. 70, 3619 (1993).

${ }^{15}$ R.G.P. van der Kraan and H. van Kempen, Surf. Sci. 338, 19 (1995).

${ }^{16}$ M. Wuttig, S. Junghans, T. Flores, and S. Blügel, Phys. Rev. B 53, 7551 (1996).

${ }^{17}$ D. Wortmann, S. Heinze, G. Bihlmayer, and S. Blügel, Phys. Rev. B 62, 2862 (2000).

${ }^{18}$ H. Ibach, M. Giesen, T. Flores, M. Wuttig, and G. Treglia, Surf. Sci. 364, 453 (1996).

${ }^{19}$ D. Sander, S. Ouazi, V.S. Stepanyuk, D. Bazhanov, and J. Kirschner, Surf. Sci. 512, 281 (2002).

${ }^{20}$ M. Cankurtaran, G.A. Saunders, P. Ray, and Q. Wang, Phys. Rev. B 47, $3161(1993), Y /(1-\nu)=\left(1+2 c_{12} / c_{11}\right)\left(c_{11}-c_{12}\right), c_{12}$ $=98 \mathrm{GPa}, c_{11}=170 \mathrm{GPa}$.

${ }^{21}$ C.P. Gazzara, R.M. Middleton, R.J. Weiss, and E.O. Hall, Acta Crystallogr. 22, 859 (1967), the atomic volume for $\mathrm{Mn}$ and $\mathrm{Cu}$ is $12.2 \AA^{3}$ and $11.8 \AA^{3}$, respectively.

${ }^{22}$ W. Pan, D. Sander, M.-T. Lin, and J. Kirschner (unpublished).

${ }^{23}$ L. Szunyogh, J. Zabloudil, P. Weinberger, F. Offi, W. Kuch, and J. Kirschner, Phys. Rev. B 67, 054418 (2003).

${ }^{24}$ F. Offi, W. Kuch, L.I. Chelaru, K. Fukumoto, M. Kotsugi, and J. Kirschner, Phys. Rev. B 67, 094419 (2003). 